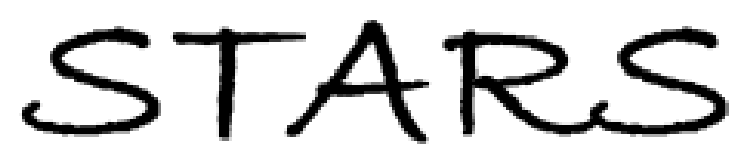

University of Central Florida

STARS

$1-1-2009$

\title{
Altering infrared metamaterial performance through metal resonance damping
}

\author{
J. Ginn \\ University of Central Florida \\ D. Shelton \\ University of Central Florida \\ P. Krenz \\ University of Central Florida \\ B. Lail \\ G. Boreman \\ University of Central Florida
}

Find similar works at: https://stars.library.ucf.edu/facultybib2000

University of Central Florida Libraries http://library.ucf.edu

This Article is brought to you for free and open access by the Faculty Bibliography at STARS. It has been accepted for inclusion in Faculty Bibliography 2000s by an authorized administrator of STARS. For more information, please contactSTARS@ucf.edu.

\section{Recommended Citation}

Ginn, J.; Shelton, D.; Krenz, P.; Lail, B.; and Boreman, G., "Altering infrared metamaterial performance through metal resonance damping" (2009). Faculty Bibliography 2000s. 1577.

https://stars.library.ucf.edu/facultybib2000/1577

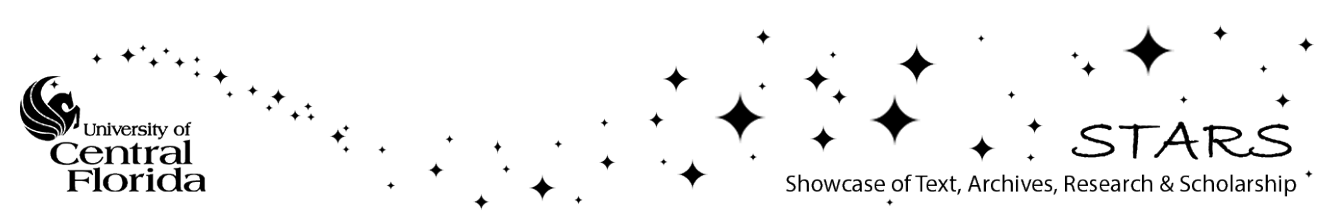




\section{Altering infrared metamaterial performance through metal resonance damping}

Cite as: J. Appl. Phys. 105, 074304 (2009); https://doi.org/10.1063/1.3093698

Submitted: 10 December 2008 . Accepted: 28 January 2009 . Published Online: 02 April 2009

J. Ginn, D. Shelton, P. Krenz, B. Lail, and G. Boreman

\section{ARTICLES YOU MAY BE INTERESTED IN}

Seebeck nanoantennas for solar energy harvesting

Applied Physics Letters 105, 093108 (2014); https://doi.org/10.1063/1.4895028

Experimental demonstration of ultrasensitive sensing with terahertz metamaterial absorbers: A comparison with the metasurfaces

Applied Physics Letters 106, 031107 (2015); https://doi.org/10.1063/1.4906109

Near- and far-field measurements of phase-ramped frequency selective surfaces at infrared wavelengths

Journal of Applied Physics 116, 044903 (2014); https://doi.org/10.1063/1.4890868

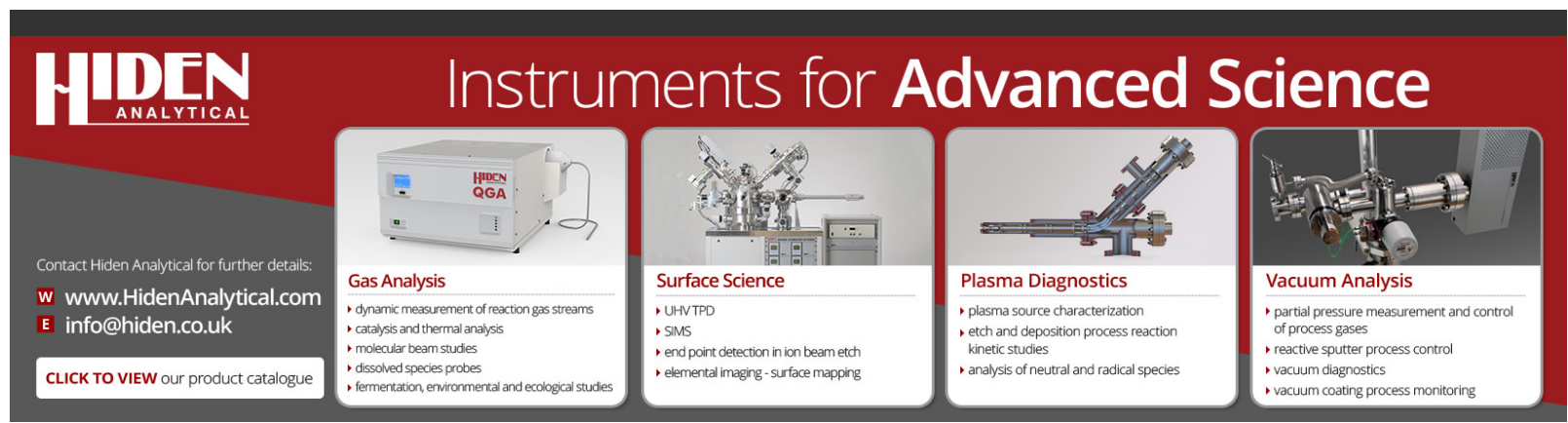




\title{
Altering infrared metamaterial performance through metal resonance damping
}

\author{
J. Ginn, ${ }^{1, a)}$ D. Shelton, ${ }^{1}$ P. Krenz, ${ }^{1}$ B. Lail, ${ }^{2}$ and G. Boreman ${ }^{1}$ \\ ${ }^{1}$ CREOL/College of Optics and Photonics, University of Central Florida, Orlando, Florida 32816, USA \\ ${ }^{2}$ Department of Electrical and Computer Engineering, Florida Institute of Technology, Melbourne, \\ Florida 32901, USA
}

(Received 10 December 2008; accepted 28 January 2009; published online 2 April 2009)

\begin{abstract}
Infrared metamaterial design is a rapidly developing field and there are increasing demands for effective optimization and tuning techniques. One approach to tuning is to alter the material properties of the metals making up the resonant metamaterial to purposefully introduce resonance frequency and bandwidth damping. Damping in the infrared portion of the spectrum is unique for metamaterials because the frequency is on the order of the inverse of the relaxation time for most noble metals. Metals with small relaxation times exhibit less resonance frequency damping over a greater portion of the infrared than metals with a longer relaxation time and, subsequently, larger dc conductivity. This leads to the unexpected condition where it is possible to select a metal that simultaneously increases a metamaterial's bandwidth and resonance frequency without altering the geometry of the structure. Starting with the classical microwave equation for thin-film resistors, a practical equivalent-circuit model is developed predicting the sensitivity of infrared metamaterials to complex film impedance. Several full-wave electromagnetic models are developed to validate the resonant-circuit model, and excellent agreement is demonstrated between modeled and measured results. (c) 2009 American Institute of Physics. [DOI: 10.1063/1.3093698]
\end{abstract}

\section{INTRODUCTION}

Over the past few years, a great deal of development has been observed in the field of infrared metamaterials. Metamaterials, in their broadest definition, are composite materials consisting of subwavelength resonant element arrays that exhibit electromagnetic properties not observed in nature. Numerous prototype devices that meet the definition of metamaterials have been successfully demonstrated at infrared frequencies including frequency selective surfaces (FSSs), ${ }^{1}$ resonant absorbers, ${ }^{2}$ reflectarrays, ${ }^{3}$ resonant polarizers, ${ }^{4}$ and negative index materials ${ }^{5}$ (NIMs) - all surfaces relying on passive, resonant antenna elements. With initial proofs of concepts in hand, it is important to begin investigation into practical techniques for selectively altering the performance of metamaterial devices in the infrared for the purpose of design optimization or tuning.

The spectral response of an infrared metamaterial is determined by four characteristics: element geometry, array spacing, substrate/superstrate material, and element material. Array spacing is usually constrained by the need to avoid the appearance of unwanted grating lobes. ${ }^{6}$ Substrate and superstrate material selection is similarly limited due to the lack of low-loss dielectric materials in the infrared band. Thus, infrared designs have typically followed the approach developed for low-frequency metamaterials and focused exclusively on altering element geometry ${ }^{7-9}$ to achieve a desired spectral response. In the microwave portion of the spectrum, it is common to tune the response of resonant structures through the introduction of passive constructs such as lumped elements or stubs ${ }^{10}$ or active elements such as

${ }^{a)}$ Electronic mail: jcginn@creol.ucf.edu. varactors $^{11}$ and optical switches. ${ }^{12}$ These are difficult approaches in the infrared due to limitations imposed by microscale lithography, material dispersion, and active-element time constants. Thus, it is of high importance to investigate any design approach that will yield element performance alteration without significant fabrication challenges.

One approach to achieve metamaterial optimization or spectral tuning in the infrared without altering the element geometry is to exploit the structure's sensitivity of the resonance behavior on the metal-film conductivity. Variation in metal conductivity alters the Ohmic loss in the metamaterial, which yields resonance damping and two phenomena that are important for design optimization: bandwidth expansion and spectral shifting. Resonance damping is a well understood concept within the physics and engineering community. If a harmonic oscillator encounters a resistive force, the system's oscillations will become damped, occurring at a lower frequency and magnitude compared to the undamped case. Consequently, the decrease in the magnitude of the oscillation must result in an increased resonance bandwidth for the system. For resonant antenna devices, the resistive force typically takes the form of attenuation due to the conversion of electromagnetic energy to heat. Passive, infrared resonant elements are especially sensitive to damping due to the highly dispersive and lossy nature of metals in the infrared band.

The effect of damping due to the finite conductivity in infrared metamaterials has been published previously, but not heavily explored as a means of metamaterial optimization or tuning. Raynolds et al. ${ }^{13}$ acknowledged that altering the conductivity of a FSS resulted in frequency shifting, attributing this phenomenon to an increase in the real part of the per- 
mittivity of the metal film with frequency. Novotny ${ }^{14}$ provided a much more detailed analysis of this behavior for visible antennas and found results similar to the ones presented in Secs. II-IV, without considering the related resonance-bandwidth issue. Frequency and bandwidth scaling due to damping can also be observed in the results of numerous other papers (such as Refs. 7 and 15), but damping effects have largely been neglected or not properly identified.

\section{METAMATERIAL DAMPING THEORY}

\section{A. Thin-film resistor impedance at infrared frequencies}

Before introducing an equivalent resonator model, it is necessary to first develop a method for determining the Ohmic loss of a metamaterial element in the infrared. For the purpose of this derivation, a simple metamaterial unit-cell element contained within an infinite length sheet of the same material with thickness $h$ is assumed to consist of a dipole slab of width $w$ and length $l$. The dipole is excited by a plane wave normally incident on the dipole's surface with an electric-field component of the form

$$
\widetilde{E}(z)=\hat{x} E_{0} e^{j \gamma z},
$$

where $\gamma$ is the propagation constant of the plane wave inside the metal film, $l$ is orientated in the $x$-direction, $w$ is orientated in the $y$-direction, and $h$ is orientated in the $z$-direction. The current density excited by the wave as it penetrates the surface is

$$
\widetilde{J}(z)=\hat{x} \sigma E_{0} e^{j \gamma z},
$$

where $\sigma$ is the bulk admittivity (dynamic complex conductivity) of the film. The propagation constant can be defined in terms of the optical properties of the metal film:

$$
\gamma=\frac{2 \pi f(n+j k)}{c},
$$

where $f$ is the frequency, $n$ is the index of refraction, $k$ is the extinction coefficient, and $c$ is the speed of light in a vacuum. The relative permeability of the metal in the infrared is assumed to be unity. The total current, I, flowing through the metal film along the length of the dipole can be found by solving the integral

$$
I=w \int_{0}^{h} \widetilde{J}(z) d z \cdot \hat{x}
$$

Neglecting the phase term of the propagation constant, Eq. (4) yields the expression

$$
I=\frac{c w \sigma E_{0}}{2 \pi f k}\left(1-e^{-2 \pi f k h / c}\right) .
$$

Equation (5) can be reduced by substituting in skin depth, $\delta_{\text {skin }}$, or

$$
\delta_{\text {skin }}=\frac{c}{2 \pi f k}
$$

$$
I=w \sigma \delta_{\text {skin }} E_{0}\left(1-e^{-h / \delta_{\text {skin }}}\right)
$$

Similarly, the voltage, $V$, developed over the length of the slab can be found by

$$
V=E_{0} l .
$$

Finally, the impedance of the film, $Z_{\text {film }}$, can be determined by Ohm's law

$$
\frac{V}{I}=Z_{\text {film }}=\frac{l}{w \delta_{\text {skin }} \sigma\left(1-e^{-h / \delta_{\text {skin }}}\right)} .
$$

Equation (9) can be recognized as the classical thin-film resistor impedance equation when the ratio of the film thickness to skin depth approaches infinity.

In the microwave portion of the spectrum, it is customary to assume that the admittivity of the metal film is purely real and nearly equal to the dc conductivity. This conclusion is from the Drude-Lorentz model, which relates dc conductivity and metal relaxation time to admittivity:

$$
\sigma(f)=\frac{\sigma_{0}}{(2 \pi f \tau)^{2}+1}(1+j 2 \pi f \tau)
$$

where $\tau$ is the metal's relaxation time and $\sigma_{0}$ is the metal's bulk dc conductivity. The relaxation time for most noble metals will be on the order of 10-100 fs, ${ }^{16}$ which causes the imaginary term in Eq. (10) to approach zero at low frequencies. In the terahertz, the imaginary term can no longer be neglected, as the frequency is on the same order as the inverse of the relaxation time. Thus, it is of importance to account for complex admittivity in Eq. (9) by relating the conductivity directly to the film's optical properties through

$$
\sigma=j 2 \pi f \varepsilon_{0}\left[1-(n+j k)^{2}\right],
$$

where $\varepsilon_{0}$ is the free-space permittivity. Substituting Eq. (11) into Eq. (9) yields

$$
Z_{\text {film }}=\frac{l}{2 \pi f \varepsilon_{0} w \delta_{\text {skin }}\left(1-e^{\left.-h / \delta_{\text {skin }}\right)}\left[2 n k+j\left(k^{2}-n^{2}+1\right)\right]\right.} .
$$

Equation (12) can also be rewritten in terms of relative permittivity, $\varepsilon_{r}$, using the relationship

$$
\varepsilon_{r}^{\prime}+j \varepsilon_{r}^{\prime \prime}=n^{2}-k^{2}+j 2 n k,
$$

which yields

$$
Z_{\text {film }}=\frac{l\left[\varepsilon_{r}^{\prime \prime}+j\left(\varepsilon_{r}^{\prime}-1\right)\right]}{2 \pi f \varepsilon_{0} w \delta_{\text {skin }}\left(1-e^{-h /} \delta_{\text {skin }}\right)\left[\left(\varepsilon_{r}^{\prime \prime}\right)^{2}+\left(1-\varepsilon_{r}^{\prime}\right)^{2}\right]} .
$$

Assuming that $\varepsilon_{r}^{\prime} \ll-1$, which is typical for metals outside of the visible and ultraviolet portion of the spectrum, ${ }^{16} \mathrm{Eq}$. (14) can be simplified and separated into complex components

$$
R_{\mathrm{film}} \approx \frac{l}{2 \pi f \varepsilon_{0} w \delta_{\text {skin }}\left(1-e^{\left.-h / \delta_{\text {skin }}\right)}\right.}\left\{\frac{\varepsilon_{r}^{\prime \prime}}{\left[\left(\varepsilon_{r}^{\prime}\right)^{2}+\left(\varepsilon_{r}^{\prime \prime}\right)^{2}\right]}\right\},
$$




$$
X_{\text {film }} \approx \frac{l}{2 \pi f \varepsilon_{0} w \delta_{\text {skin }}\left(1-e^{-h /} \delta_{\text {skin }}\right)}\left\{\frac{\varepsilon_{r}^{\prime}}{\left[\left(\varepsilon_{r}^{\prime}\right)^{2}+\left(\varepsilon_{r}^{\prime \prime}\right)^{2}\right]}\right\},
$$

where $R_{\text {film }}$ is the resistivity of the film and $X_{\text {film }}$ is the reactivity of the film. From Eqs. (15) and (16), the ratio of the resistive and reactive components of the metal film is nearly equal to the material's loss tangent, $\tan (\delta)$, or

$$
\frac{R_{\mathrm{film}}}{X_{\mathrm{film}}} \approx \tan (\delta) \equiv \frac{\varepsilon_{r}^{\prime \prime}}{\varepsilon_{r}^{\prime}} .
$$

Finally, the expressions for $R_{\text {film }}$ and $X_{\text {film }}$ can be further simplified when they are expressed in terms of loss tangent and the real part of permittivity:

$$
\begin{aligned}
& R_{\text {film }} \approx \frac{l}{2 \pi f \varepsilon_{0} w \delta_{\text {skin }}\left(1-e^{-h / \delta_{\text {skin }}}\right)}\left\{\frac{\tan (\delta)}{\varepsilon_{r}^{\prime}\left[1+\tan (\delta)^{2}\right]}\right\}, \\
& X_{\text {film }} \approx \frac{R_{\text {film }}}{\tan (\delta)} .
\end{aligned}
$$

The values of $R_{\text {film }}$ and $X_{\text {film }}$ represent the complex impedance of the metal slab presented to the incident radiation. Unlike the situation in the microwave band, the slab is no longer purely resistive. It will exhibit a largely capacitive response, because $\varepsilon_{r}^{\prime}$ is strongly negative in the infrared, with a loss tangent approaching unity.

\section{B. Equivalent-circuit resonator derivation}

Using the dipole layout presented in Sec. II A, an equivalent circuit for an infinite array of this element at primary resonance is presented in Fig. 1. The resonance properties of the equivalent circuit can be expressed by

$$
\begin{aligned}
& \Delta f=\frac{R}{2 \pi L}, \\
& f=\frac{1}{2} \sqrt{\frac{1}{\pi^{2} L C}-(\Delta f)^{2}},
\end{aligned}
$$

where $\Delta f$ is the full width at half-power (FWHP) bandwidth of the dipole, $R$ is the effective resistance of the dipole observed by a current excited at the surface of the dipole, $L$ is the effective inductance of the slab, and $C$ is the effective capacitance. Inductance and capacitance are predominantly determined by the geometries of the element and the unit cell, with the resistance determined by the loss of the metal making up the dipole. The impedance of the dipole can be determined by summing in series the structural, or resonant, impedance of the element, assuming no damping or metal loss, and the impedance of the metal slab calculated previously. The resistance of the equivalent circuit can be found by adding in series the film's resistance and the structural resistance, $R_{0}$, of the dipole:

$$
R=R_{\text {film }}+R_{0} .
$$

The structural resistance is caused by selective loading, substrate loss, or unwanted reradiation. Similarly, the inductance

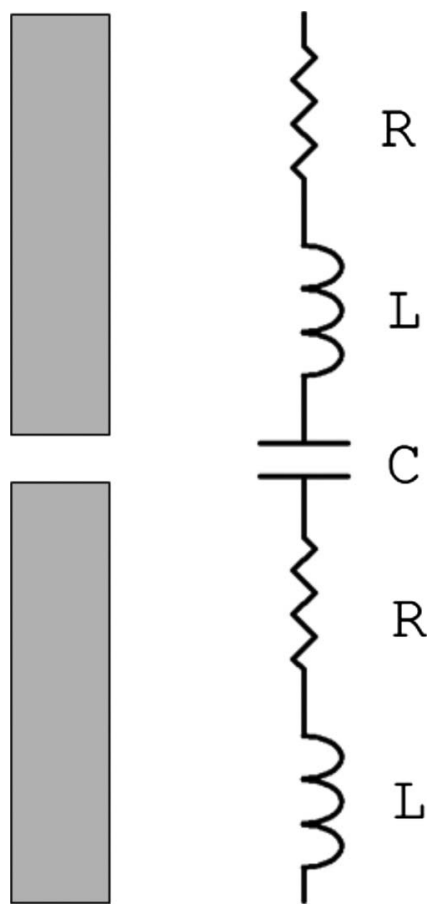

FIG. 1. Resonant circuit equivalent model for an infinite array of dipole metamaterial elements. Mutual coupling is assumed to only occur along the direction of the length of the dipole.

of the equivalent circuit can be related to the reactance of the metal film through

$$
L=L_{0}-\frac{1}{2 \pi f X_{\text {film }}},
$$

where $L_{0}$ is the structural inductance. Capacitance is assumed to be independent of the metal-film properties and is entirely determined by

$$
C=C_{0},
$$

where $C_{0}$ is the structural capacitance due to mutual coupling.

Several important conclusions can be made regarding damping in the infrared using the proposed equivalent-circuit model. As expected, increasing the loss of the metal will result in an increase in the dipole's resonance bandwidth from Eq. (20) and this increase in bandwidth will result in an increase in the resonance frequency from Eq. (21). Interestingly, increasing the magnitude of the reactance of the film will further increase the resonance frequency by decreasing the inductance of the slab from Eqs. (23) and (21). The reactance of the film has a limited impact on the bandwidth of the system relative to the resistance of the film because $R_{0}$ will be significantly smaller than $R_{\text {film }}$ for any good metamaterial structure, with most antenna loss occurring due to the metal making up the resonator. This leads to the unique situation where it is possible to have a narrowband design, with a small $R_{\text {film }}$, experience a significant decrease in the resonance frequency due to having a simultaneously large $X_{\text {film }}$. The specific conditions required for this to occur, in terms of metal properties, are discussed later.

Unfortunately, there are several limitations inherent to this theory and design approach. Metals in the infrared, un- 


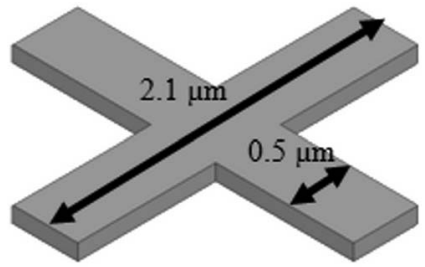

FIG. 2. Dimensions of cross metamaterial element.

like the microwave portion of the spectrum, exhibit appreciable dispersion. The development of these equivalentcircuit models assumes quasistatic parameter values which will lead to errors in the predicted bandwidths of the resonant metamaterial. The presented model also does not provide methods for accounting for some nonidealities present in the metal films, such as the anomalous skin effect and film depth profile variations. Most of these limitations can be mitigated by employing some form of full-wave electromagnetic modeling, which will be discussed in Sec. III. In general, optimization using metamaterial damping is not suitable for every application since it involves increasing the absorption loss of the metamaterial element. Additional film absorption will lead to unwanted heat loading on the metamaterial element which may lead to structural or performance issues.

\section{VALIDATION OF THEORY}

For validation of the proposed equivalent-circuit model, an infinite array of cross FSS elements was modeled at normal incidence using ANSOFT HFSS, a finite-element-method electromagnetic solver. Cross elements were chosen due to their wide use in metamaterial structures, their similarity in performance to dipole elements, and their polarization insensitivity at normal incidence. The dimensions of the element considered are presented in Fig. 2, and the element's square unit-cell size was fixed at $2.5 \mu \mathrm{m}$. The thickness of the metal film was fixed at $100 \mathrm{~nm}$. The superstrate is taken as vacuum $\left(\varepsilon_{r}=1\right)$ and the substrate as lossless silicon $\left(\varepsilon_{r}\right.$ =11.4). This design was chosen with typical dimensions for resonance near the long-wave infrared (LWIR) $\mathrm{CO}_{2}$ laser line at $10.6 \mu \mathrm{m}$ or $28.28 \mathrm{THz}$.

\section{A. Simulation}

\section{Real frequency-independent conductivity}

The proposed element was first modeled with four frequency-independent real values of conductivity typical for the infrared $(\sigma=0.5,1,5,10 \mathrm{MS} / \mathrm{m})$. In addition, the modeled performance of the design using a perfect electric conductor (PEC) material $(\sigma=\infty \mathrm{S} / \mathrm{m})$ was found to determine the undamped response. Unless the option to solve inside of a metal film is explicitly enabled, HFSS applies a finiteconductivity boundary to the metal-film surface, where the thickness of the film is assumed to be infinite and the admittivity is assumed to be purely real for the calculation of surface impedance. ${ }^{17}$ The finite-conductivity boundary surface condition is used to investigate the sensitivity of the crosses' bandwidth and resonance frequency to changes in conductiv-

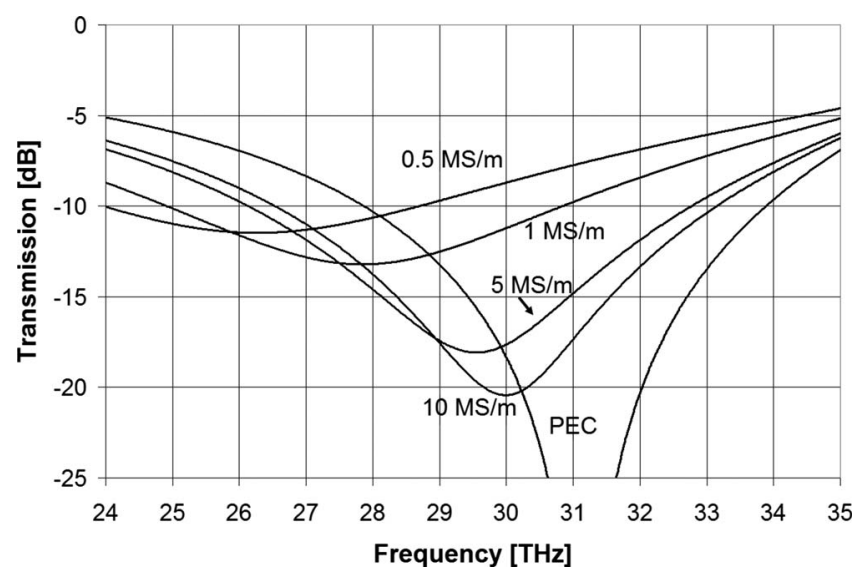

FIG. 3. Modeled transmission of the cross element with four frequencyindependent conductivities.

ity without influences of unwanted radiation propagation through the film or finite skin depth. Modeled results are presented in Fig. 3. As expected from Eqs. (9), (18), and (19), the modeled cross metamaterial exhibits increased damping with decreasing conductivity. Because the conductivity is real, no reactive components are present to influence the damping behavior.

\section{Complex frequency-independent impedance surface}

To study how the complex impedance of the film impacts resonance behavior and to validate the claims used to develop Eqs. (23) and (24), the cross design was modeled using four complex film impedances. HFSS has the capability to model surface boundaries with complex sheet resistance; however, the surface must have zero thickness, limiting the practicality of this approach. Sheet resistance can be related to the film impedance through

$$
Z_{\text {film-sheet }}=\frac{w}{l} Z_{\text {film }} \text {. }
$$

The complex sheet resistance values used in modeling are presented in Table I, as well as modeled center frequency and bandwidth. Each model is labeled with the format $\left(R_{\text {film-sheet }}, X_{\text {film-sheet }}\right)$, and the modeled results are presented in Fig. 4. As expected, reducing the resistance of the film reduced the bandwidth and increased the resonance frequency. Similarly, increasing the reactance of the metal film resulted in a decrease in the resonance frequency. Slight variations in bandwidth are also observed with changes in reactance, but the bandwidth is significantly more sensitive

TABLE I. Frequency-independent sheet resistances used in modeling and modeled resonance frequency and bandwidth.

\begin{tabular}{ccccc}
\hline \hline Model name & $\begin{array}{c}R_{\text {film-sheet }} \\
(\Omega / \square)\end{array}$ & $\begin{array}{c}X_{\text {film-sheet }} \\
(\Omega / \square)\end{array}$ & $\begin{array}{c}f \\
(\mathrm{THz})\end{array}$ & $\begin{array}{c}\Delta f \\
(\mathrm{THz})\end{array}$ \\
\hline$(1,1)$ & 1 & 1 & 28.10 & 1.46 \\
$(1,2)$ & 1 & 2 & 27.46 & 1.29 \\
$(2,1)$ & 2 & 1 & 27.95 & 2.82 \\
$(2,2)$ & 2 & 2 & 27.32 & 2.58 \\
\hline \hline
\end{tabular}




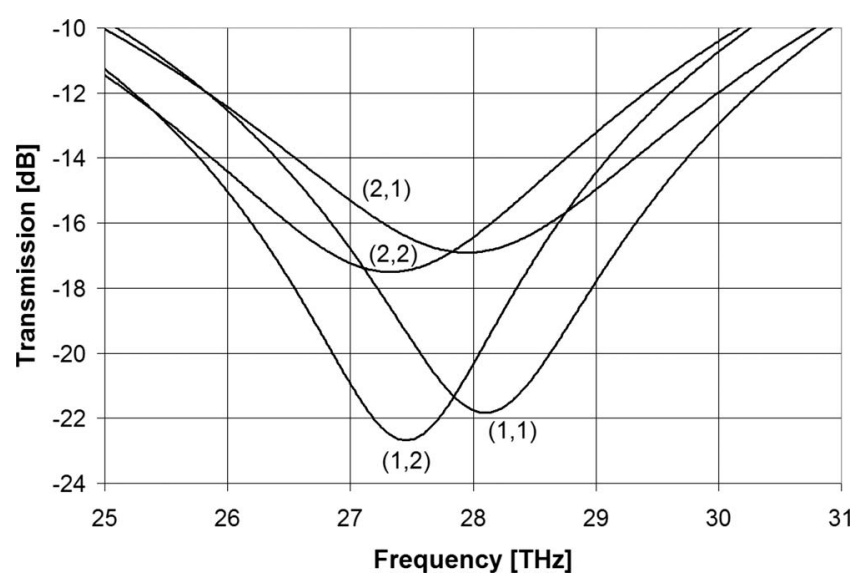

FIG. 4. Modeled transmission of the cross element with four frequencyindependent complex sheet resistances.

to the resistance of the film, validating the claim in Sec. III A 1.

\section{Complex frequency-independent conductivity}

It is also important to consider a more realistic model to test the validity of Eqs. (18) and (19). Thus, the cross from Fig. 2 was remodeled using representative frequencyindependent complex dielectric parameters, and HFSS was instructed to solve inside the metal films. Four individual models were compared to determine the crosses' change in response due to changes in magnitude of $\varepsilon_{r}^{\prime}, \tan (\delta)$, and $\delta_{\text {skin }}$. The skin depth can be found directly from permittivity and loss tangent using the relationship

$$
\delta_{\text {skin }}=\frac{c}{\pi f \sqrt{2\left(\left|\varepsilon_{r}^{\prime}\right| \sqrt{1+\tan (\delta)^{2}}-\varepsilon_{r}^{\prime}\right)}} .
$$

The permittivity values used and the derived parameters are listed in Table II. Each model is labeled using a multiple of permittivity and loss tangent relative to those used in the first model with the format $\left(\varepsilon_{r}^{\prime}, \tan (\delta)\right)$. The modeled results are presented in Fig. 5.

The results presented in Fig. 5 are consistent with the equivalent-circuit model and Eqs. (15) and (16)_increasing the magnitude of the real part of permittivity decreases bandwidth and increasing the magnitude of the imaginary part of permittivity counteracts damping and shifts the resonance to higher frequencies. One of the more intriguing results is that model $(2,0.5)$ demonstrates a narrower bandwidth than model $(2,1)$, even though both models have the same real part of permittivity and model $(2,1)$ resonates at a higher frequency. This is caused by the loss tangent of the metal

TABLE II. Frequency-independent permittivities used in modeling and derived loss tangent and skin depth values.

\begin{tabular}{ccccc}
\hline \hline Model name & $\varepsilon_{r}^{\prime}$ & $\varepsilon_{r}^{\prime \prime}$ & $\tan \delta$ & $(28.28 \mathrm{THz}, \mathrm{nm})$ \\
\hline$(1,1)$ & -1800 & 2880 & -1.6 & 33.12 \\
$(2,0.5)$ & -3600 & 2880 & -0.8 & 26.98 \\
$(1,2)$ & -1800 & 5760 & -3.2 & 25.76 \\
$(2,1)$ & -3600 & 5760 & -1.6 & 23.42 \\
\hline \hline
\end{tabular}

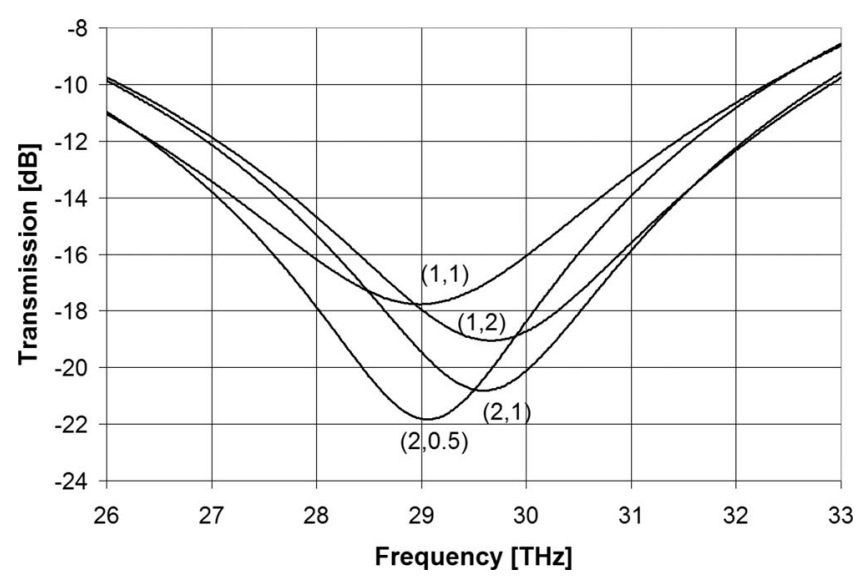

FIG. 5. Modeled transmission of the cross element with four frequencyindependent complex permittivities.

also playing a role in determining the resistance of the metal film. Minimizing $\tan (\delta)$ and maximizing the magnitude of $\varepsilon_{r}^{\prime}$, from Eq. (18), will yield a smaller film resistance and, thus, a smaller bandwidth. Minimizing $\tan (\delta)$, however, also increases the reactive component of the metal film resulting in a larger resonance frequency. Conversely, $(1,2)$ has a narrower bandwidth than $(1,1)$ due to a larger loss tangent, which yields a lower film resistance, but the smaller $\varepsilon_{r}^{\prime}$ results in a larger overall bandwidth when compared to $(2,0.5)$ or $(2,1)$.

\section{Measured metal-film properties}

To further test the proposed theory, the cross design was modeled using measured-metal permittivities, which were complex and frequency dependent. Three metals were selected for comparison due to their distinct properties in the LWIR: gold for a loss tangent magnitude less than 1, aluminum for a loss tangent magnitude greater than 1 , and nickel for a loss tangent magnitude nearly equal to 1 . Determination of the optical properties of these metals in the infrared was done using films evaporated on a silicon witness sample and a J.A. Woollam IR-VASE ellipsometer. ${ }^{18}$ The measured and derived optical properties are presented in Figs. 6-8. The modeled resonance behaviors of the crosses using the measured optical properties of the metals are presented in Fig. 9.

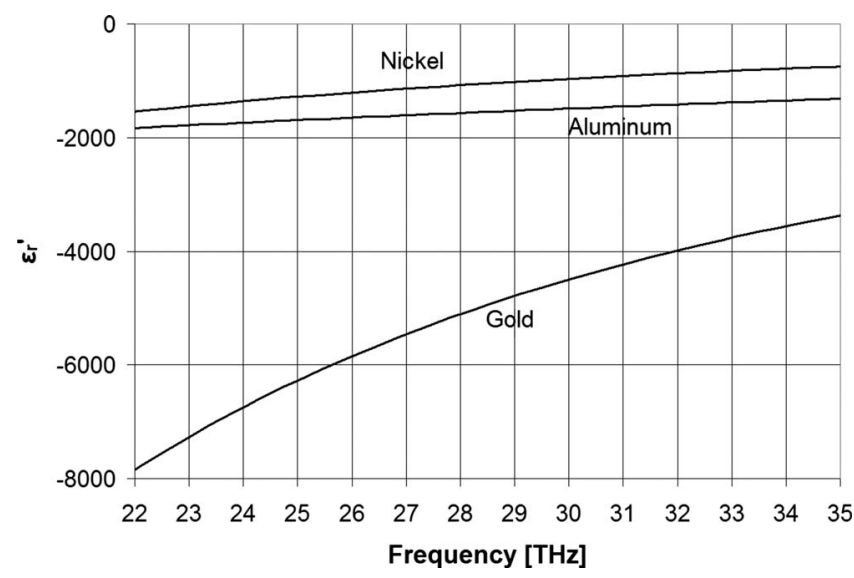

FIG. 6. Measured real part of permittivity for gold, aluminum, and nickel. 


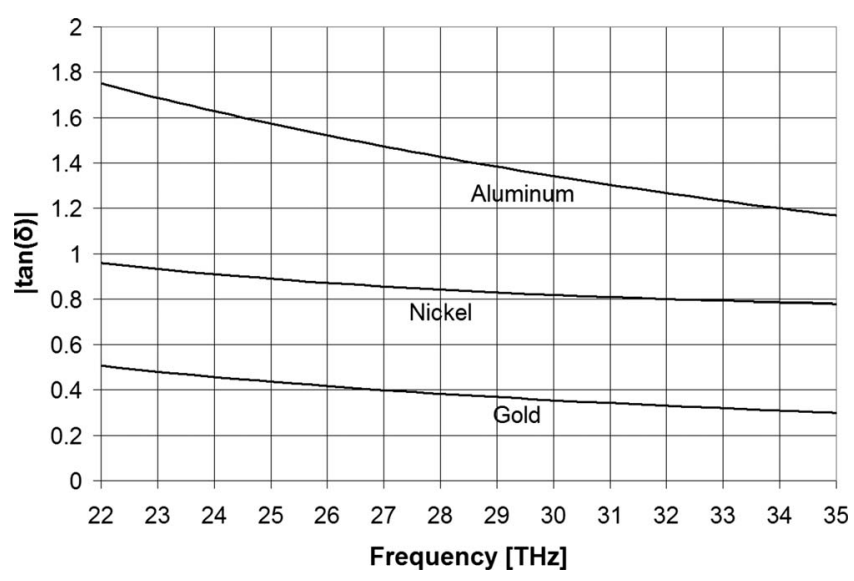

FIG. 7. Measured loss tangent for gold, aluminum, and nickel.

Once again, the results are consistent with the predictions from the equivalent-circuit model. Nickel demonstrates the most damped response since its magnitude of the real part of permittivity is small and its loss tangent is nearly equal to 1. Gold has the largest magnitude of the real part of permittivity of the three metals and, thus, the lowest film loss and therefore exhibits the narrowest bandwidth and deepest notch. Consistent with results from the frequencyindependent simulation, aluminum's large loss tangent magnitude decreases the film's reactive component and allows it to resonate at the highest frequency, closest to the undamped design. However, aluminum's small real part of permittivity results in a notch significantly shallower than gold, as well as a larger bandwidth.

\section{B. Measured validation}

To validate the modeled results, the three metal cross prototypes were fabricated and measured. Fabrication followed the electron-beam lithography process outlined in Ref. 19. Deposition of the metal films was done using electronbeam evaporation for gold and nickel and thermal evaporation for aluminum. The substrate was a $350-\mu$ m-thick highresistivity, double-side-polished silicon wafer. A scanning electron microscope (SEM) micrograph of a portion of the fabricated array is presented in Fig. 10. Measurement of the transmission of these surfaces was carried out using a

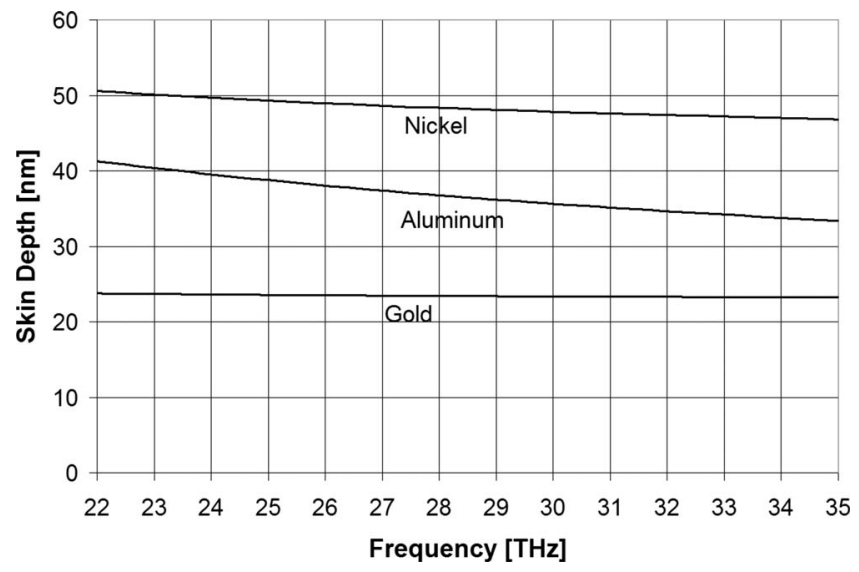

FIG. 8. Calculated skin depth for gold, aluminum, and nickel.

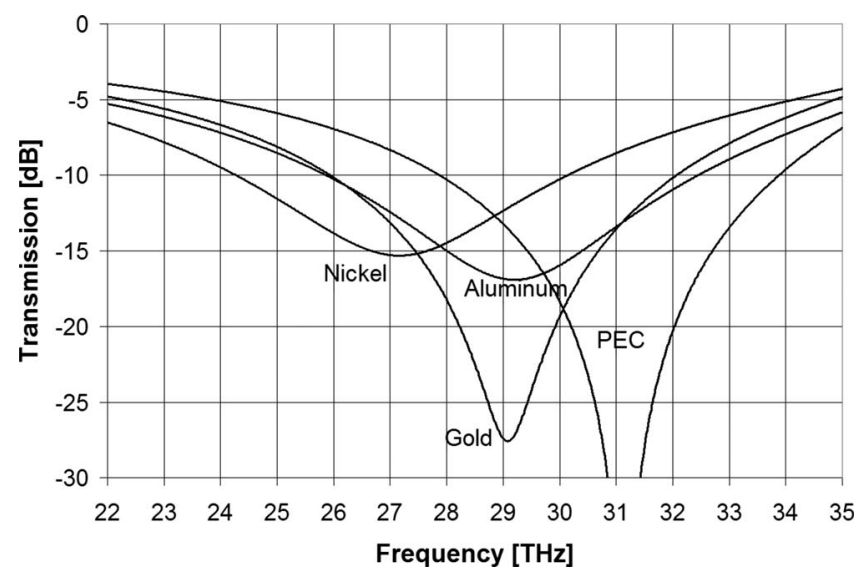

FIG. 9. Modeled transmission of four cross metamaterial arrays made of three different metals and PEC.

Perkin-Elmer micro-Fourier transform infrared (FTIR) spectrometer with a microscope attachment. Results from this measurement, as well as the modeled results from Fig. 9, are compared in Fig. 11. Both modeling and profilometer measurements indicated that the thickness of the gold film was approximately $90 \mathrm{~nm}$, while the other films were approximately $100 \mathrm{~nm}$ thick.

Overall, excellent agreement is observed between modeled and measured results. Ringing is present in all of the measured results due to the Fabry-Pérot resonance from the finite thickness of the substrate. This also leads to slight differences between modeled and measured notch depths since the HFSS model neglects the backside reflection. Additional differences can be attributed to the presence of native oxide layers for the nickel and aluminum films as well as the likely presence of residual resist.

\section{DISCUSSION}

\section{A. Impact of relaxation time on damping and film reactance}

Based on the circuit model and measured results it is clear, with films sufficiently thick compared to the skin depth, that the loss tangent and the real part of permittivity are the best indicator of metamaterial resonance performance. From Eqs. (10), (11), and (13), loss tangent can be defined completely in terms of metal relaxation time, or

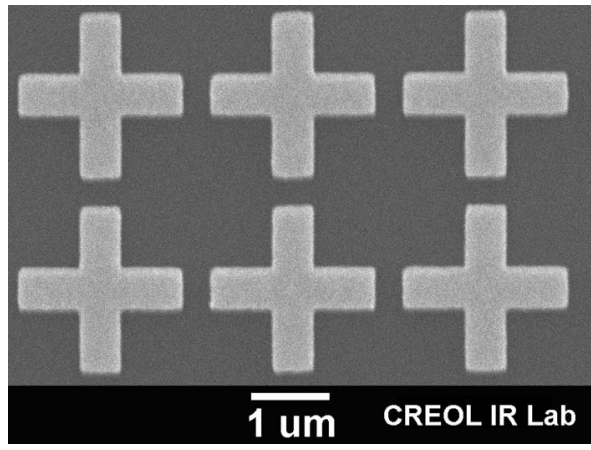

FIG. 10. SEM micrograph of a portion of the gold cross metamaterial array. 


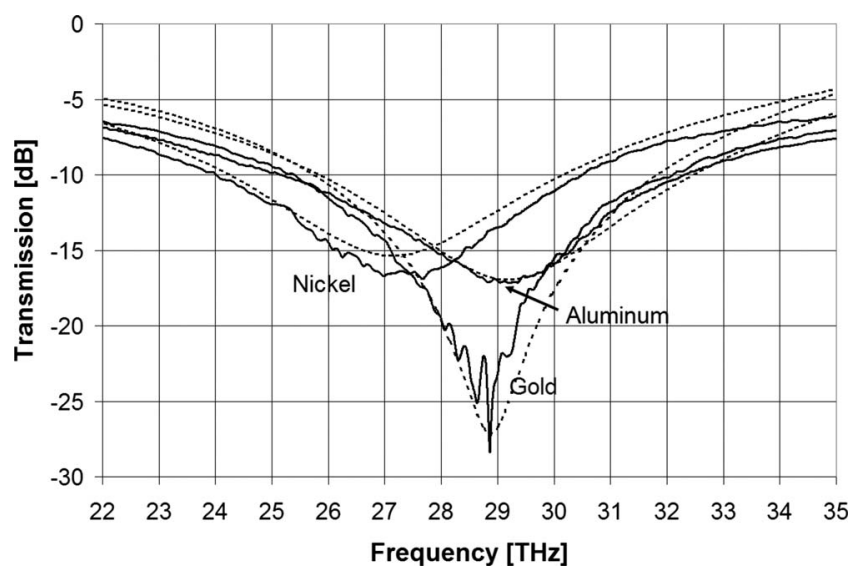

FIG. 11. Measured (solid) and modeled (dotted) transmissions of three cross metamaterial arrays made up of three different metals.

$$
\tan (\delta) \approx \frac{-1}{2 \pi f \tau},
$$

again assuming that $\left|\varepsilon_{r}^{\prime}\right| \gg 1$. Similarly, the real part of permittivity can also be defined in terms of loss tangent:

$$
\varepsilon_{r}^{\prime} \approx \frac{N e^{2}}{m_{0} \varepsilon_{0}(2 \pi f)^{2}}\left[\frac{-1}{1+\tan (\delta)^{2}}\right] .
$$

With the usual definition of dc conductivity,

$$
\sigma_{0}=\frac{N e^{2} \tau}{m_{0}},
$$

where $N$ is the carrier density, $e$ is the electronic charge constant, and $m_{0}$ is the electron rest mass. Substituting Eqs. (27) and (28) into Eqs. (18), (19), and (26) yields

$$
\begin{aligned}
& X_{\text {film }} \approx \frac{-m_{0} 2 \pi f l}{N e^{2} w \delta_{\text {skin }}}, \\
& R_{\text {film }} \approx-X_{\text {film }}(2 \pi f \tau)^{-1}, \\
& \delta_{\text {skin }} \approx \frac{c \sqrt{1+\left(\frac{1}{2 \pi f \tau}\right)^{2}}}{\sqrt{\frac{N e^{2}}{2 m_{0} \varepsilon_{0}}\left(1+\sqrt{1+\left(\frac{1}{2 \pi f \tau}\right)^{2}}\right)}},
\end{aligned}
$$

assuming an infinite film thickness for simplicity. From the previous results, to minimize bandwidth damping, it is desirable to maximize relaxation time, while reducing film resistivity. Conversely, to counteract frequency damping by decreasing the reactance of the film, it is desirable to minimize relaxation time, resulting in a larger skin depth. The only other option available to a metamaterial designer is to alter the carrier density which can be achieved by utilizing different metals and not necessarily through film processing. Of the two, metal-film relaxation time provides the largest range of variation through the alteration of impurity scattering. ${ }^{20}$

It should also be noted that this type of design optimization is limited to frequencies close to the relaxation frequency, or $(2 \pi \tau)^{-1}$. For most metals, the relaxation frequency falls in the infrared and terahertz frequency bands. To

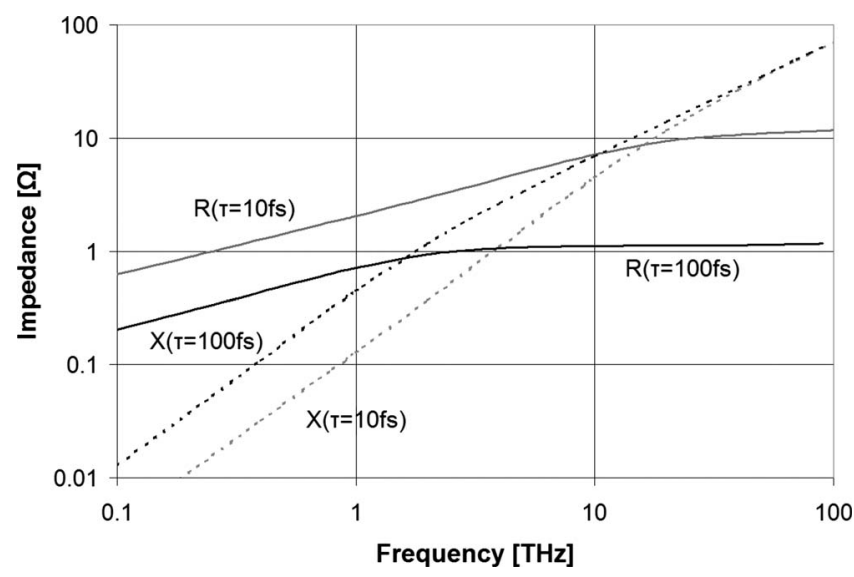

FIG. 12. The complex impedance of two bulk metals with a relaxation time of 10 or $100 \mathrm{fs}$ in the infrared and terahertz. Carrier concentration for the two metals is assumed to be the same.

illustrate, the complex impedances of two fictitious bulk metals with relaxation times equal to 10 and 100 fs are plotted in Fig. 12. Both metals have the same carrier concentration and their dc conductivity is set to $10 \exp (20) \tau \mathrm{S} / \mathrm{m} \mathrm{s}$. From the figure and earlier results, it is not possible to counteract damping for these two metals in the microwave portion of the spectrum by reducing the reactivity of the metal film because the impedance is already nearly real. Above the relaxation frequency and into the visible, the real part of the impedance for both metals has approached its maximum value and the reactive component becomes dominant and nearly the same for all relaxation times. Thus, in both the microwave and visible, the metal with the highest dc conductivity, and typically the largest relaxation time, will have the narrowest bandwidth and highest resonance frequency.

The region of interest for the proposed optimization approach falls in between the relaxation frequencies of the two metals. In this region, the metal with a higher real part of impedance also has the smaller reactive component. Assuming that an appropriate geometry is chosen to exploit the reduced reactivity of the film, according to Eq. (21) or similar expression, it is possible to have a metamaterial structure resonate at a higher frequency with a lossy metal than with a metal having a larger dc conductivity. The metal film's higher resistivity will still yield a larger bandwidth than the metal with the larger dc conductivity, unless the carrier concentration is significantly different.

\section{B. Bandwidth considerations}

In the development of the equivalent-circuit model, the bandwidth of the metamaterial was defined as the FWHP bandwidth about the resonance frequency. This definition is meaningful for devices where notch depth is significant, such as phased devices like reflectarrays or devices where a strong resonance is necessary to approximate an equivalent material response, such as negative permittivity in NIM metamaterials. For band-reject filter designs, like the cross metamaterial characterized in Sec. IV A, the actual depth of the notch is not necessarily important, as long as the transmission of the reject band is below some arbitrary floor value. Thus, it has been customary in this situation to define the bandwidth rela- 
TABLE III. Calculated bandwidths for the three measured-metal designs in Fig. 9

\begin{tabular}{lccc}
\hline \hline Metal & $\begin{array}{c}f \\
(\mathrm{THz})\end{array}$ & $\begin{array}{c}\Delta f \\
(\mathrm{THz})\end{array}$ & $\begin{array}{c}3 \mathrm{~dB} \Delta f \\
(\mathrm{THz})\end{array}$ \\
\hline Gold & 29.09 & 0.77 & 9.71 \\
Aluminum & 29.21 & 3.23 & 10.29 \\
Nickel & 27.16 & 3.77 & 9.50 \\
\hline \hline
\end{tabular}

tive to the two $3 \mathrm{~dB}$ roll-off points below the filter's passband and not relative to the center resonance frequency.

To investigate the impact of damping on the $3 \mathrm{~dB}$ bandwidth, the two sets of bandwidths for the modeled results in Fig. 9 were calculated and are presented in Table III. As expected from the equivalent-circuit model, the FWHP bandwidth increased as the metal loss increased. On the other hand, the results for nickel demonstrate that damping can minimize the $3 \mathrm{~dB}$ roll-off bandwidth by reducing the contrast between the loss and passbands. This result suggests that in infrared filter applications the narrowest bandwidth can be achieved through the use of lossy metals and not necessarily through high conductivity. The trade-off for this approach is higher loss in the passband and the requirement of smaller element dimensions to counteract frequency damping.

\section{Additional applications}

The applications of resonance damping are not just limited to filter applications. Infrared emission surfaces typically utilize lossy metals ${ }^{21}$ to increase bandwidth and have fewer issues with incident coupling efficiency, from the presence of a resonant cavity. These surfaces can especially benefit from resonance damping for fine tuning of spectral performance without the limitations present in transmissive surfaces. Similarly, extremely narrowband metamaterials, such as electron bandgap devices, can utilize damping to fine tune their response, even at a postfabrication stage, using etching or polishing. Similar results have also been seen for antennacoupled bolometer designs in the infrared, ${ }^{22}$ where the antennas effectively behaved as uncoupled resonant elements.

The results from this study have implications for tunable infrared metamaterials as well. Due to the short time constant needed for varactor operation in the infrared, ${ }^{23}$ future nonmechanical tuning devices in the infrared will most likely behave as a varistor element. Based on the results presented, using a varistor to increase the overall resistance of the metamaterial will be able to tune the center frequency from the ideal resonance frequency, however, with an undesirable increase in bandwidth. Optical switches will face a similar limitation unless their on-state has conductivity on the order of the metal making up the metamaterial.

\section{CONCLUSIONS AND FUTURE WORK}

In this work, a new approach to metamaterial optimization is proposed. By altering the relaxation time of the metal film making up a metamaterial, the performance of the metamaterial will correspondingly change. It has been demonstrated that properly designed metamaterials consisting of metals with small relaxation times exhibit less frequency damping in the infrared than do designs with larger relaxation times and larger dc conductivities. This leads to the unexpected situation where a metamaterial design can be altered to simultaneously resonate at higher frequencies with larger bandwidths. This unique result is verified with an analytical circuit model, full-wave modeling, and measurement. Future work will include investigating parameter extraction and alternative geometries.

\section{ACKNOWLEDGMENTS}

The authors would like to thank Dr. Jeff Tharp, Ansoft Corporation, and Dr. Javier Alda, University Complutense of Madrid, for their helpful discussions.

${ }^{1}$ C. M. Rhoades, E. K. Damon, and B. A. Munk, Appl. Opt. 21, 2814 (1982)

2I. Pusçasu, W. Schaich, and G. Boreman, Infrared Phys. Technol. 43, 101 (2002).

${ }^{3}$ J. Ginn, B. Lail, J. Alda, and G. Boreman, Opt. Lett. 33, 779 (2008)

${ }^{4}$ J. Tharp, J. López-Alonso, J. Ginn, C. Middleton, B. Lail, B. Munk, and G. Boreman, Opt. Lett. 31, 2687 (2006).

${ }^{5}$ S. Zhang, W. Fan, N. C. Panoiu, K. J. Malloy, R. M. Osgood, and S. R. J. Brueck, Phys. Rev. Lett. 95, 137404 (2005).

${ }^{6}$ B. Munk, Frequency Selective Surfaces: Theory and Design (Wiley, New York, 2000), p. 23.

${ }^{7}$ I. Pusçasu, W. Schaich, and G. Boreman, Appl. Opt. 40, 118 (2001).

${ }^{8}$ J. A. Bossard, D. H. Werner, T. S. Mayer, J. A. Smith, Y. Tang, R. P. Drupp, and L. Li, IEEE Trans. Antennas Propag. 54, 1265 (2006).

${ }^{9}$ A. Kildishev, U. Chettiar, Z. Liu, V. Shalaev, D. Kwon, Z. Bayraktar, and D. Werner, J. Opt. Soc. Am. B 24, A34 (2007).

${ }^{10}$ B. Munk, R. Kouyoumjian, and L. Peters, IEEE Trans. Antennas Propag. 19, 612 (1971).

${ }^{11}$ T. Chang, R. Langley, and E. Parker, IEE Proc. Microwaves, Antennas Propag. 143, 62 (1996).

${ }^{12}$ M. Chaharmir, J. Shaker, M. Cuhaci, and A. Sebak, IEEE Trans. Antennas Propag. 54, 1134 (2006).

${ }^{13}$ J. Raynolds, B. Munk, J. Pryor, and R. Marhefka, J. Appl. Phys. 93, 5346 (2003).

${ }^{14}$ L. Novotny, Phys. Rev. Lett. 98, 266802 (2007).

${ }^{15}$ C. Fumeaux, M. Gritz, I. Codreanu, W. Schaich, F. González, and G. Boreman, Infrared Phys. Technol. 41, 271 (2000).

${ }^{16}$ M. Fox, Optical Properties of Solids (Oxford University Press, Oxford, 2003), pp. 145-150.

${ }^{17}$ Ansoft HFSS Reference Manual, Rel. 10, Ansoft Corporation, 2005.

${ }^{18}$ J. Ginn, B. Lail, D. Shelton, J. Tharp, W. Folks, and G. Boreman, Appl. Comput. Electromagn. Soc. J. 22, 184 (2007).

${ }^{19}$ J. Ginn, B. Lail, and G. Boreman, IEEE Trans. Antennas Propag. 55, 2989 (2007).

${ }^{20}$ D. Shelton, T. Sun, J. Ginn, K. Coffey, and G. Boreman, J. Appl. Phys. 104, 103514 (2008)

${ }^{21}$ B. Monacelli, J. Pryor, B. A. Munk, D. Kotter, and G. Boreman, IEEE Trans. Antennas Propag. 53, 745 (2005).

${ }^{22}$ F. González, J. Alda, J. Simón, J. Ginn, and G. Boreman, Infrared Phys. Technol. 52, 48 (2009).

${ }^{23}$ H. Kazemi, K. Shinohara, G. Nagy, W. Ha, B. Lail, E. Grossman, G. Zummo, W. Folks, J. Alda, and G. Boreman, Proc. SPIE 6542, 65421J-1 (2007). 